\title{
Determining MRI Inflammation Targets When Considering a Rheumatoid Arthritis Treat-to-Target Strategy: Results of a Randomized, Placebo-Controlled Trial
}

\author{
Harris A. Ahmad (1) - Joshua F. Baker · Mikkel Østergaard · \\ June Ye $\cdot$ Paul Emery $\cdot$ Philip G. Conaghan
}

Received: February 25, 2019 / Published online: July 5, 2019

(C) The Author(s) 2019

\begin{abstract}
Introduction: Magnetic resonance imaging (MRI) is increasingly used in patients with rheumatoid arthritis (RA) to determine residual inflammation after treatment and as a predictor
\end{abstract}

Enhanced Digital Features To view enhanced digital features for this article go to https://doi.org/10.6084/ m9.figshare.8289821.

Electronic Supplementary Material The online version of this article (https://doi.org/10.1007/s12325019-01020-6) contains supplementary material, which is available to authorized users.

H. A. Ahmad $(\bowtie) \cdot$ J. Ye

Bristol-Myers Squibb, Princeton, NJ, USA

e-mail: harris.ahmad@bms.com

\section{J. F. Baker}

Philadelphia VA Medical Center, University of

Pennsylvania, Philadelphia, PA, USA

M. Østergaard

Copenhagen Center for Arthritis Research, Center for Rheumatology and Spine Diseases,

Rigshospitalet, Glostrup, Copenhagen, Denmark

M. Østergaard

Department of Clinical Medicine, University of

Copenhagen, Copenhagen, Denmark

P. Emery · P. G. Conaghan

Leeds Institute of Rheumatic and Musculoskeletal

Medicine, University of Leeds, Leeds, UK

P. Emery · P. G. Conaghan

NIHR Leeds Biomedical Research Centre, Leeds

Teaching Hospitals NHS Trust, Leeds, UK of structural damage progression. Establishing an optimal threshold of inflammatory activity that predicts lower risk of structural damage progression may inform treatment decisions. This post hoc analysis investigated whether patients with RA at low risk of structural damage progression can be identified based on MRI inflammation thresholds.

Methods: Hand and wrist MRI was performed at baseline, and at months 6 and 12 in a phase $3 \mathrm{~b}$, randomized, active-controlled, double-blind trial of abatacept in early RA (AVERT). Pathologies were scored using the OMERACT RA MRI Score. Data were stratified into two risk subgroups (less and more severe inflammation) for structural damage progression (erosion change $>0.5$ ) based on baseline inflammation. In this post hoc analysis, log odds ratios of probability of progression \{adjusted for baseline Disease Activity Score in 28 joints [C-reactive protein; DAS28 (CRP)]\} were compared between subgroups to test the performance of inflammation thresholds.

Results: There were 351 randomized and treated patients with baseline MRIs, of whom 276 (78.6\%) and $235(67.0 \%)$ had MRIs available at months 6 and 12, respectively. The DAS28 (CRP)-adjusted probabilities of progression from baseline to month 12 based on scores at baseline, and from months 6 to 12 based on month 6 scores, were significantly lower among patients with less inflammation $(P<0.0001-0.0459)$, independent of clinical 
disease activity. Predefined thresholds of synovitis $\leq 3$ (total score 21 ), osteitis $\leq 3$ (total score 69) and total inflammation score (osteitis double-weighted) $\leq 9$ were associated with a lower likelihood of structural damage progression in unadjusted analyses.

Conclusion: Levels of MRI-determined inflammatory activity below defined thresholds were independently associated with a lower risk of structural damage progression in early RA, providing a potential trial endpoint for levels of inflammation not associated with progression.

Trial Registration: ClinicalTrials.gov identifier, NCT01142726.

Funding: Bristol-Myers Squibb.

Keywords: Biological therapies; DMARDs; Inflammation; Magnetic resonance imaging; Rheumatoid arthritis; Rhuematology

\section{INTRODUCTION}

The concept of treat-to-target is now well established in the management of rheumatoid arthritis (RA), with clinical targets aimed at low disease activity or remission [1]. Modern imaging, including magnetic resonance imaging (MRI) and ultrasonography, is increasingly used to accurately determine residual inflammation after treatment, as imaging-detected inflammation can predict structural damage progression [2-6]. However, there is little information on how low inflammatory activity must be on imaging in order to be confident that progression will not occur [5]. Additionally, treating physicians face a challenge when determining to what extent inflammation can be managed with symptomatic treatments alone or when more potent agents, such as biologic diseasemodifying antirheumatic drugs (DMARDs), are required.

A previous study suggested levels of synovitis, osteitis and a combined total inflammation score correlated with the risk of structural damage progression independent of clinical disease activity [7]. Validation of these levels would provide physicians with clinically relevant, imaging-detected inflammation targets. Establishing a threshold of inflammatory activity that predicts a lower risk of structural damage progression would help to avoid unnecessary initiation, premature tapering or other changes to treatment, as well as inform the physician on whether a review of treatment was needed. The aim of this post hoc analysis was to examine the outcomes of patients with early RA participating in a large clinical trial, based on their degree of MRI-determined synovitis, osteitis and combined total inflammatory activity, and to validate the aforementioned approach as a predictive trial outcome for non-progression.

\section{METHODS}

\section{Study Design and Participants}

Assessing Very Early Rheumatoid arthritis Treatment (AVERT) was a phase 3b, randomized, active-controlled, 24-month trial in patients with early RA (ClinicalTrials.gov identifier: NCT01142726) [8]. The trial included a 12-month, double-blind treatment period in which patients received abatacept plus methotrexate (MTX), abatacept monotherapy or MTX alone (supplementary Fig. 1). Subcutaneous abatacept was administered at $125 \mathrm{mg} /$ week. MTX was titrated to $15-20 \mathrm{mg} /$ week within 6-8 weeks. The study design and results have been described previously $[8,9]$. The AVERT study protocol was approved by the Institutional Review Board or Independent Ethics Committee at each site. All patients provided written informed consent and the study was performed in accordance with the Declaration of Helsinki.

Contrast-enhanced 1.5-T MRIs of the clinically most active hand and wrist were performed [9]. Inflammation (synovitis and osteitis) and erosions were scored at baseline, and at months 6 and 12 by two independent central radiologists (Charles Peterfy and Yan Chen of Spire Sciences) blinded to treatment arm and chronological order, using the Outcome Measures in Rheumatology Clinical Trials (OMERACT) RA MRI Score (RAMRIS) method [10]. The scores of the two readers were 
averaged and the top 5\% of discrepancies were adjudicated by consensus review [9].

\section{MRI Inflammation Thresholds Analysis and Structural Damage Progression}

MRI data were pooled from all three treatment arms for this post hoc analysis. Patients were stratified into two risk subgroups (less and more severe inflammation) based on MRI inflammation scores at the start of the observation period. The thresholds for less severe inflammation (based on preliminary reported levels) were: $\leq 3$ for synovitis (total score 21) $\leq 3$ for osteitis (total score 69) and $\leq 9$ when combined. For combined total inflammation scores, osteitis was double-weighted due to its greater ability in multivariate analysis to predict structural damage progression and its greater effect on subsequent erosion development compared with synovitis, as assessed by radiography and MRI $[7,11]$. Structural damage progression was defined as erosion change from baseline $>0.5$ (OMERACT-RAMRIS erosion score scale for each bone: 0-10; range of scores: 0-230) [12] and greater than the smallest detectable change (SDC) (SDC value was 2.29) [9]. In order to provide a comprehensive analysis, structural damage progression was analyzed using MRI inflammation scores at various time points: 0-12 months by MRI scores at baseline, and 6-12 months by MRI scores at month 6 .

\section{Statistical Analyses}

Risks of progression (erosion change from baseline $>0.5$ and $>$ SDC) were compared between subgroups and treatment groups using baseline Disease Activity Score in 28 joints [Creactive protein; DAS28 (CRP)]-adjusted logistic models. Odds ratios and related $P$ values were reported.

The linear relationship between unadjusted predicted probability of progression and erosion score $(>0.5)$ at baseline was explored ('as-observed' analysis).

\section{RESULTS}

\section{Patient Disposition and Baseline Demographic Data}

A total of 351 patients with early RA were randomized, treated and had MRI data at baseline [9]. Of these patients, $276(78.6 \%)$ and 235 (67.0\%) also had MRI data available at month 6 and month 12, respectively. Baseline demographic data and clinical characteristics for the overall trial population [8] and MRI population [9] have been reported previously. Briefly, patients were anti-cyclic citrullinated peptide-2 positive, had a mean (SD) age of 47.0 (12.6) years and symptom duration of $0.56(0.50)$ years, with $77.8 \%$ being female [8]. Patients had highly active disease [mean (SD) tender joint count of 13.6 (7.7) and swollen joint count of 11.1 (7.1)] with indicators of poor prognosis (rheumatoid factor positivity: 95.2\%; mean CRP: $17.5 \mathrm{mg} / \mathrm{L})$.

\section{MRI Inflammation Thresholds and Structural Damage Progression}

For the overall population, when adjusted for baseline DAS28 (CRP), the risks of structural damage progression (defined as $>0.5$ or $>$ SDC) at follow-up was significantly lower in the subgroup with less versus more severe inflammation at baseline for all comparisons ( $P$ value range $=<0.001-0.0459)$ (Fig. 1 ; supplementary Fig. 2). Only total inflammation score was not significantly associated with progression greater than the SDC between month 6 and month 12, where rates were very low (based on month 6 scores, $P=0.1284)$. In by-treatment analyses [also adjusted for baseline DAS28 (CRP)], patients with less severe inflammation at baseline showed significantly less progression from 0 to 12 months than patients with more severe inflammation in most comparisons across all treatment groups (supplementary Tables 1 and 2 ). The above observations were similar when data were not adjusted for clinical activity, as determined by DAS28 (CRP) (supplementary Tables 3 and 4). 


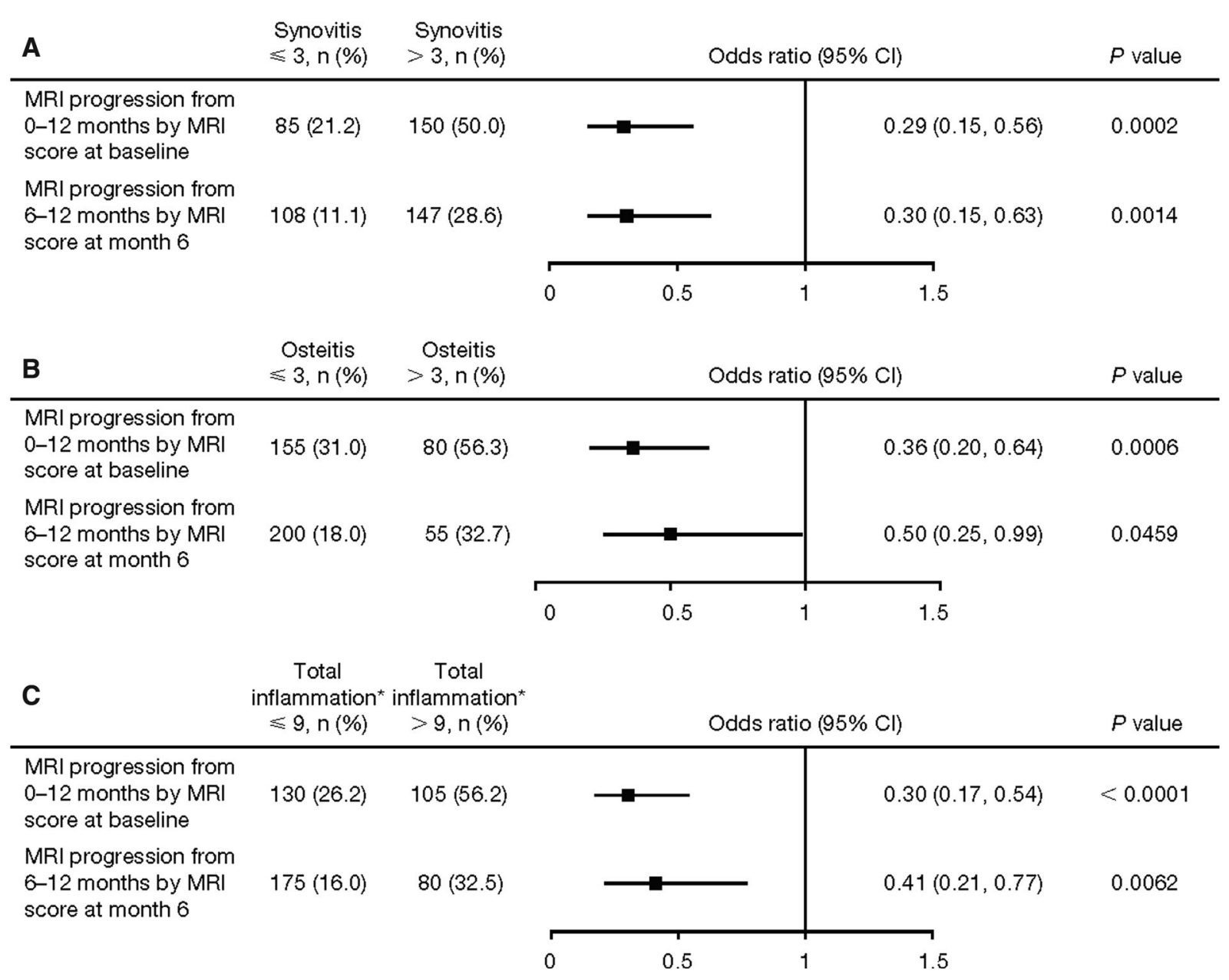

Fig. 1 Percentage and baseline DAS28 (CRP)-adjusted odds ratios of MRI structural progression $(>0.5)$ in the overall population from 0 to 12 months by MRI inflammation scores (a synovitis, b osteitis and $\mathbf{c}$ total inflammation) at baseline ('as-observed' population). *Total

\section{MRI Inflammation Threshold Analysis}

The unadjusted probability of progression (defined as $>0.5$ ) from baseline to month 12 was lower among patients with less versus more severe inflammation at baseline (Fig. 2a). The unadjusted probability of progression from month 6 to month 12 was lower among patients with less versus more severe inflammation at month 6 (Fig. 2b). Similar trends were seen for the unadjusted log odds progression analysis (supplementary Fig. 3). inflammation is synovitis + osteitis double-weighted. $C I$ confidence interval, DAS28 (CRP) disease activity score in 28 joints (C-reactive protein), MRI magnetic resonance imaging

Fig. 2 Association between inflammation scores and unadjusted predicted probability of MRI structural progression ('as-observed' population). a Baseline inflammation scores and MRI progression (defined as change from 0 to 12 months in erosion score $>0.5)$; vertical dotted lines denote osteitis/synovitis score $\leq 3$ or total inflammation score $\leq 9$ at baseline;.b Month 6 inflammation scores and MRI progression (defined as change from 6 to 12 months in erosion score $>0.5)$; vertical dotted lines denote osteitis/synovitis score $\leq 3$ or total inflammation score $\leq 9$ at month 6. MRI magnetic resonance imaging 

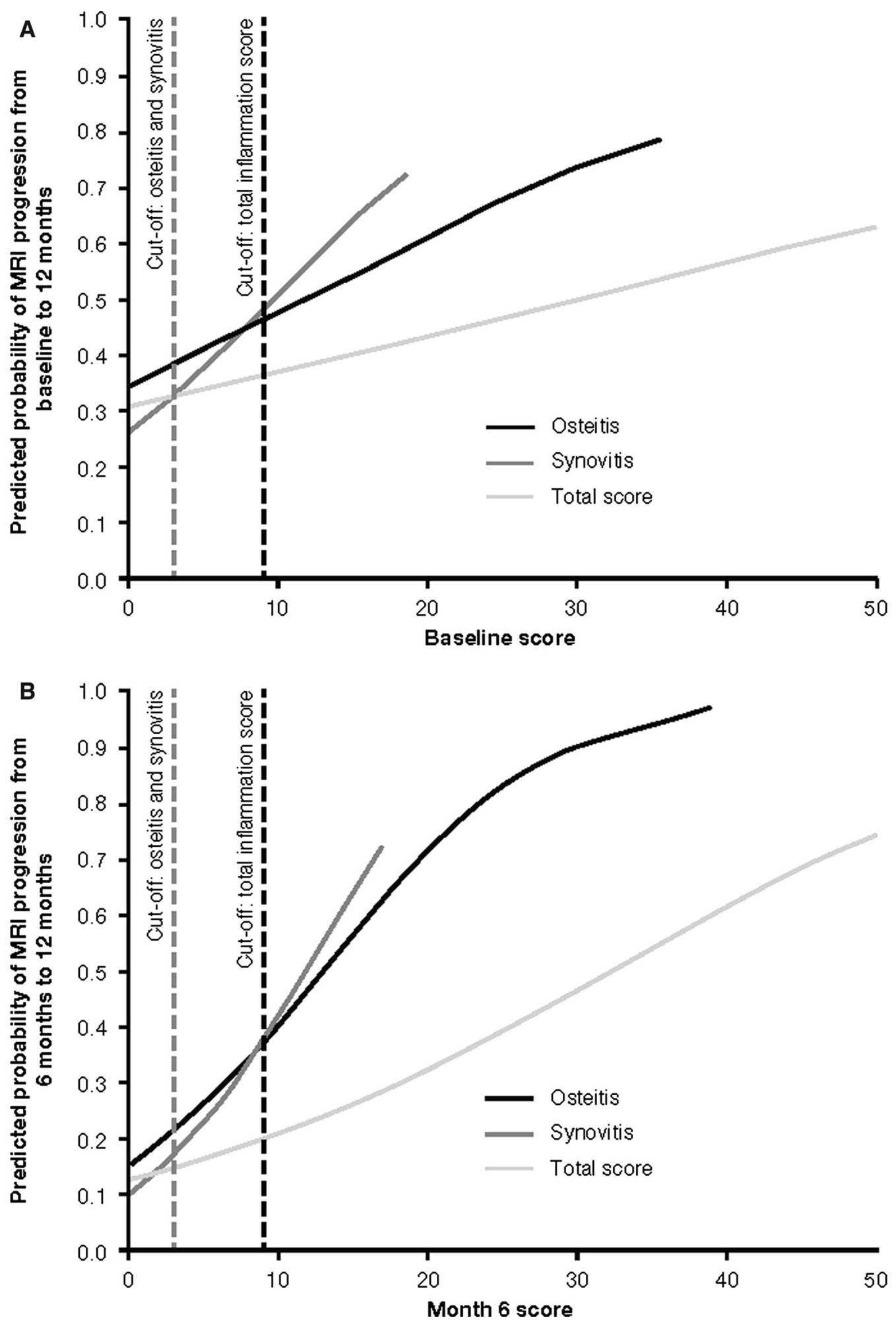


\section{DISCUSSION}

This analysis established that lower levels of MRI inflammation below the predefined thresholds $[\leq 3$ for synovitis, $\leq 3$ for osteitis and $\leq 9$ (osteitis double-weighted) when combined into a total inflammation score] were associated with a very low risk of MRI-detected, baseline DAS28 (CRP)-adjusted structural damage progression.

MRI is increasingly being used as an outcome measure in RA clinical trials, usually in patients with at least moderate disease activity. MollerBisgaard et al. recently reported a randomized clinical trial (IMAGINE-RA) targeting the absence of MRI osteitis versus a clinical treat-totarget approach in patients with RA in clinical remission; no improvement of remission or radiographic progression rates versus the conventional strategy were observed [13]. However, the IMAGINE-RA trial contrasts with the current study with regard to trial design, baseline level of disease and patient populations. For example, in the current study, baseline mean DAS28CRP score [8] ranged from 5.3 to 5.5 and osteitis scores ranged from 2.5 to 5.1, whereas in IMAGINE-RA, baseline median DAS28-CRP and MRI osteitis scores were 1.9/2.0 and 2.0/2.0 (for both arms, respectively) [13]. Furthermore, the IMAGINE-RA study targeted absence of osteitis, whereas the present study investigates a combined osteitis and synovitis target. Consequently, future trials with treat-to-target endpoints could benefit from including combined MRI thresholds as a primary study outcome measure for joint inflammation, thereby enabling the identification of individuals with a significant burden of inflammatory disease. However, very little is currently known about what level of inflammation may be deemed acceptable (i.e., suggesting that the risk of subsequent damage is low). Subsequently, there will be a need to translate these findings into a readily usable score for routine clinical use. Inflammation score assessments, such as those presented here, may complement standard descriptive or clinical assessments and be used to assess treatment efficacy and to improve the efficacy of clinical studies. Treatment with an effective treat-to-target strategy has been shown to decrease MRI inflammation scores at 6 and 12 months, and has been associated with significant reductions in clinical disease activity and an absence of structural damage progression at 12 months [14].

The work reported here validates inflammatory thresholds defined in earlier research using golimumab trial data: data from the GOBEFORE trial were used to develop the thresholds, while GO-FORWARD data were used for validation [7]. The current validation was achieved despite differences across the trial populations: patients in the GO-BEFORE trial were MTX-naïve (as in AVERT) [8, 15], whereas those in GO-FORWARD had an inadequate response to MTX [16]. Differences between the golimumab and AVERT trials also included the drug and treatment regimens $[8,15,16]$, and patients in AVERT had early RA (persistent symptoms for $\leq 2$ years) [8], whereas those in the golimumab trials had a longer-standing disease $[15,16]$.

In a GO-FORWARD validation analysis, $4.7 \%$ of patients with less severe total inflammation (i.e., no greater than the threshold of 9) versus $29.6 \%$ of patients with more severe total inflammation (i.e., greater than the threshold of 9) at month 6 had structural damage progression between months 6 and 12 (progression defined as $>0.5$; odds ratio: 0.11) [7]. In the current analysis, these figures were $16.0 \%$ with less severe versus $32.5 \%$ with more severe total inflammation [baseline DAS28 (CRP)-adjusted odds ratio: 0.41]. Overall, progression rates were higher in the current study compared with the golimumab analyses, likely due to the selection of only patients who were seropositive and had highly active disease. To identify an appropriate cut-off for change in MRI, an erosion change of $>0.5$, which has been described as a good discriminator of radiography-detected structural progression [12], and SDC, which has been reported to be less variable and enable smaller differences in disease progression to be detected [17], were used in this study. Despite these differences in overall rates of structural damage progression, our analysis supports previous evidence that a low MRI inflammation score is 
associated with an approximately 80\% lower odds of structural damage progression.

In a GO-FORWARD validation analysis of the defined thresholds for synovitis and osteitis, respectively, $7.8 \%$ and $9.0 \%$ of patients with less severe inflammation (i.e., no greater than the thresholds of 3) at month 6 had structural damage progression by month 12 [7]. In the current analysis, these figures were $11.1 \%$ and $18.0 \%$, respectively. The importance of MRI inflammation thresholds regarding subsequent radiographic progression has been documented in previous studies $[6,11,12,18,19]$.

Strengths of the current analysis include the large trial size and the well-defined study population of MTX-naïve, anti-cyclic citrullinated peptide-positive patients with highly active, early RA. This research also validates earlier work in a distinct patient population treated with a different agent.

Limitations of this analysis, which should be considered when interpreting data, include that, as the study focused on patients with highly active early RA, the findings may not be generalizable to other populations. The work is also a post hoc analysis, with inherent limitations, such as the thresholds described here were not tested in the trial as a pre-specified outcome measure and the relationships between probability of progression and erosion score were from 'as-observed' analyses. Radiographic data were not available for this study; however, short-term changes in MRI-detected inflammation have been found to predict longterm changes on subsequent radiography in RA [20]. A limitation of defining low inflammatory thresholds is that older patients and those with osteoarthritis may have detectable inflammation by MRI [21]. However, patients would be very unlikely to have scores above the threshold used in this current study; for example, the median synovitis score reported was 0.5 with an inter-quartile range of $0-2$, suggesting $75 \%$ of patients from in the general population had scores $\leq 2[21]$.

Further research is needed to determine whether these and other thresholds, such as those including tenosynovitis, on MRI are applicable and optimal across different populations of patients with RA and during treatment with DMARDs with different mechanisms of action. Additionally, an investigation into whether achievement of low MRI-detected inflammation is associated with optimization of patient-reported outcomes would be very timely $[21,22]$ and could form the basis for a logical next study.

\section{CONCLUSION}

In summary, this post hoc analysis indicates that the achievement of target levels of inflammation in patients with early RA is associated with a very low risk of structural damage progression. These post hoc data provide further support for the value of measuring joint inflammation and damage using objective imaging techniques, and indicate that such measures may have the potential to be used as trial endpoints in certain patient populations and to contribute to personalized RA treatment in the future.

\section{ACKNOWLEDGEMENTS}

The authors would like to thank Dr Charles Peterfy and Dr Yan Chen of Spire Sciences for assessing the MRI data. P.E. and P.G.C. receive support from the National Institute for Health Research (NIHR) Leeds Biomedical Research Centre. The views expressed are those of the author(s) and not necessarily those of the National Health Service, the NIHR or the Department of Health. The authors also thank the participants of the study, as well as Yedid Elbez for his interpretation of the data.

Funding. Sponsorship for this study, the Rapid Service Fee and the open access fee were funded by Bristol-Myers Squibb, Princeton, NJ, USA. All authors had full access to all of the data in this study and take complete responsibility for the integrity of the data and accuracy of the data analysis.

Medical Writing and Editorial Assistance. Professional medical writing and editorial assistance was provided by Carolyn Tubby, 
PhD, and Fiona Boswell, PhD, at Caudex and was funded by Bristol-Myers Squibb.

Authorship. All named authors meet the International Committee of Medical Journal Editors (ICMJE) criteria for authorship for this article, take responsibility for the integrity of the work as a whole, and have given their approval for this version to be published.

Authorship Contributions. Harris A. Ahmad., Joshua F. Baker and Mikkel Østergaard made substantial contributions to the conception or design of the work, analysis and interpretation of data. June Ye made substantial contributions to the analysis and interpretation of data. Paul Emery and Philip G Conaghan made substantial contributions to the conception or design of the work, acquisition of data, analysis and interpretation of data. All authors were responsible for drafting of the manuscript or revising it critically for important intellectual content and agree to be accountable for all aspects of the work in ensuring that questions related to the accuracy or integrity of any part of the work are appropriately investigated and resolved.

Disclosures. Harris A. Ahmad is a shareholder and employee of Bristol-Myers Squibb. June Ye is a shareholder and employee of Bristol-Myers Squibb. Joshua F. Baker has nothing to disclose. Mikkel Østergaard has received grant/research support from AbbVie, BristolMyers Squibb, Janssen and Merck, and has participated in speakers' bureaus for AbbVie, Bristol-Myers Squibb, Boehringer-Ingelheim, Celgene, Centocor, Eli Lilly, GSK, Hospira, Janssen, Merck, Mundipharma, Novartis, Novo Nordisk, Orion, Pfizer, Roche, Regeneron, Schering-Plough, Takeda, UCB and Wyeth. Paul Emery has received grant/research support from AbbVie, Merck, Pfizer and Roche, and is a consultant for AbbVie, Bristol-Myers Squibb, Lilly, Merck, Novartis, Pfizer, Roche, and Samsung Bioepis. Philip G. Conaghan is a consultant for AbbVie, Lilly, Novartis and Pfizer, and has participated in speakers' bureaus for AbbVie, Bristol-Myers Squibb and Roche.
Compliance with Ethics Guidelines. The AVERT study protocol was approved by the Institutional Review Board or Independent Ethics Committee at each site. All patients provided written informed consent and the study was performed in accordance with the Declaration of Helsinki.

Data Availability. All data generated or analyzed during this study are included in this published article and its supplementary information files.

Open Access. This article is distributed under the terms of the Creative Commons Attribution-NonCommercial 4.0 International License (http://creativecommons.org/licenses/ by-nc/4.0/), which permits any noncommercial use, distribution, and reproduction in any medium, provided you give appropriate credit to the original author(s) and the source, provide a link to the Creative Commons license, and indicate if changes were made.

\section{REFERENCES}

1. Smolen JS. Treat-to-target as an approach in inflammatory arthritis. Curr Opin Rheumatol. 2016;28(3):297-302.

2. Brown AK, Conaghan PG, Karim Z, Quinn MA, Ikeda K, Peterfy CG, et al. An explanation for the apparent dissociation between clinical remission and continued structural deterioration in rheumatoid arthritis. Arthritis Rheum. 2008;58(10):2958-67.

3. Brown AK, Quinn MA, Karim Z, Conaghan PG, Peterfy CG, Hensor E, et al. Presence of significant synovitis in rheumatoid arthritis patients with disease-modifying antirheumatic drug-induced clinical remission: evidence from an imaging study may explain structural progression. Arthritis Rheum. 2006;54(12):3761-73.

4. McQueen FM, McHaffie A, Clarke A, Lee AC, Reeves $\mathrm{Q}$, Curteis B, et al. MRI osteitis predicts cartilage damage at the wrist in RA: a three-year prospective 3T MRI study examining cartilage damage. Arthritis Res Ther. 2014;16(1):R33.

5. Gandjbakhch F, Haavardsholm EA, Conaghan PG, Ejbjerg B, Foltz V, Brown AK, et al. Determining a 
magnetic resonance imaging inflammatory activity acceptable state without subsequent radiographic progression in rheumatoid arthritis: results from a followup MRI study of 254 patients in clinical remission or low disease activity. J Rheumatol. 2014;41(2):398-406.

6. Hetland ML, Stengaard-Pedersen $\mathrm{K}$, Junker $\mathrm{P}$, Ostergaard M, Ejbjerg BJ, Jacobsen S, et al. Radiographic progression and remission rates in early rheumatoid arthritis-MRI bone oedema and antiCCP predicted radiographic progression in the 5 -year extension of the double-blind randomised CIMESTRA trial. Ann Rheum Dis. 2010;69(10):1789-95.

7. Baker JF, Østergaard M, Emery P, Baker DG, Conaghan PG. Development and validation of rheumatoid arthritis magnetic resonance imaging inflammation thresholds associated with lack of damage progression. Clin Exp Rheumatol. 2017;35(4):607-13.

8. Emery P, Burmester GR, Bykerk VP, Combe BG, Furst DE, Barre E, et al. Evaluating drug-free remission with abatacept in early rheumatoid arthritis: results from the phase $3 \mathrm{~b}$, multicentre, randomised, active-controlled AVERT study of 24 months, with a 12-month, double-blind treatment period. Ann Rheum Dis. 2015;74(1):19-26.

9. Peterfy C, Burmester GR, Bykerk VP, Combe BG, DiCarlo JC, Furst DE, et al. Sustained improvements in MRI outcomes with abatacept following the withdrawal of all treatments in patients with early, progressive rheumatoid arthritis. Ann Rheum Dis. 2016;75(8):1501-5.

10. Ostergaard M, Peterfy C, Conaghan P, McQueen F, Bird P, Ejbjerg B, et al. OMERACT Rheumatoid Arthritis Magnetic Resonance Imaging Studies. Core set of MRI acquisitions, joint pathology definitions, and the OMERACT RA-MRI scoring system. J Rheumatol. 2003;30(6):1385-6.

11. Nieuwenhuis WP, van Steenbergen HW, Stomp W, Stijnen T, Huizinga TW, Bloem JL, et al. The course of bone marrow edema in early undifferentiated arthritis and rheumatoid arthritis: a longitudinal magnetic resonance imaging study at bone level. Arthritis Rheumatol. 2016;68(5):1080-8.

12. Baker JF, Ostergaard M, Emery P, Hsia EC, Lu J, Baker DG, et al. Early MRI measures independently predict 1-year and 2-year radiographic progression in rheumatoid arthritis: secondary analysis from a large clinical trial. Ann Rheum Dis. 2014;73(11):1968-74.

13. Moller-Bisgaard S, Horslev-Petersen K, Ejbjerg B, Hetland ML, Ornbjerg LM, Glinatsi D, et al. Effect of magnetic resonance imaging vs conventional treat- to-target strategies on disease activity remission and radiographic progression in rheumatoid arthritis: the IMAGINE-RA randomized clinical trial. JAMA. 2019;321(5):461-72.

14. Axelsen MB, Eshed I, Horslev-Petersen K, StengaardPedersen K, Hetland ML, Moller J, et al. A treat-totarget strategy with methotrexate and intra-articular triamcinolone with or without adalimumab effectively reduces MRI synovitis, osteitis and tenosynovitis and halts structural damage progression in early rheumatoid arthritis: results from the OPERA randomised controlled trial. Ann Rheum Dis. $2015 ; 74(5): 867-75$.

15. Emery P, Fleischmann RM, Moreland LW, Hsia EC, Strusberg I, Durez P, et al. Golimumab, a human anti-tumor necrosis factor alpha monoclonal antibody, injected subcutaneously every four weeks in methotrexate-naive patients with active rheumatoid arthritis: twenty-four-week results of a phase III, multicenter, randomized, double-blind, placebo-controlled study of golimumab before methotrexate as first-line therapy for early-onset rheumatoid arthritis. Arthritis Rheum. 2009;60(8):2272-83.

16. Keystone EC, Genovese MC, Klareskog L, Hsia EC, Hall ST, Miranda PC, et al. Golimumab, a human antibody to tumour necrosis factor alpha given by monthly subcutaneous injections, in active rheumatoid arthritis despite methotrexate therapy: the GO-FORWARD Study. Ann Rheum Dis. 2009;68(6):789-96.

17. Bruynesteyn $K$, Boers $M$, Kostense $P$, van der Linden $S$, van der Heijde D. Deciding on progression of joint damage in paired films of individual patients: smallest detectable difference or change. Ann Rheum Dis. 2005;64(2):179-82.

18. Haavardsholm EA, Boyesen P, Ostergaard M, Schildvold A, Kvien TK. Magnetic resonance imaging findings in 84 patients with early rheumatoid arthritis: bone marrow oedema predicts erosive progression. Ann Rheum Dis. 2008;67(6):794-800.

19. Hetland ML, Ejbjerg B, Horslev-Petersen K, Jacobsen S, Vestergaard A, Jurik AG, et al. MRI bone oedema is the strongest predictor of subsequent radiographic progression in early rheumatoid arthritis. Results from a 2-year randomised controlled trial (CIMESTRA). Ann Rheum Dis. 2009;68(3):384-90.

20. Peterfy C, Strand V, Tian L, Ostergaard M, Lu Y, Dicarlo J, et al. Short-term changes on MRI predict long-term changes on radiography in rheumatoid arthritis: an analysis by an OMERACT Task Force of pooled data from four randomised controlled trials. Ann Rheum Dis. 2017;76(6):992-7. 
21. Baker JF, Conaghan PG, Emery P, Baker DG, Ostergaard M. Relationship of patient-reported outcomes with MRI measures in rheumatoid arthritis. Ann Rheum Dis. 2017;76(3):486-90.
22. Ranganath VK, Strand V. Importance of 'meeting of the minds': patient-reported outcomes and MRI. Ann Rheum Dis. 2017;76(3):473-5. 